\title{
Arbor
}

\section{Norma y ruptura en el personaje del Gracioso}

\author{
César Oliva
}

Arbor CLXXVII, 699-700 (Marzo-Abril 2004), 439-453 pp.

El presente artículo intenta ofrecer nuevos datos sobre la identidad escénica de un personaje tan arquetípico en la escena española como el gracioso. Su enfoque procede de la práctica teatral, incluyendo, pues, una revisión por las compañías y actores más representativos del siglo XX, que define de manera precisa los rasgos más característicos de dicho personaje. En cuanto al gracioso propiamente dicho cabe decir que sus máscaras o rostros proceden no sólo de la entidad literaria del personaje sino de la personalidad del actor que lo interpreta. De ahí que, a pesar del canon establecido que lo define de manera precisa, el gracioso puede ofrecer alternativas muy interesantes a su peculiar modelo, algunas de las cuales se repasan aqui cumplidamente. Por esa variedad podemos ratificar la inmensa riqueza de la comedia áurea, de la que el gracioso es oportuno ejemplo.

\section{De la entidad escénica del gracioso}

Cuando había tradición teatral en España (que haberla, la había), los papeles de gracioso los interpretaba un tipo de actor muy determinado. Yo mismo he alcanzado a ver, en los Festivales de España, al gran Manuel Dicenta haciendo el Tello de El Caballero de Olmedo, y a Antonio Ferrandis, el Cosme de La dama duende. Así mismo, tuve la suerte de dirigir a José Luis Heredia en una producción de El Burlador de Sevilla, interpretando un gran Catalinón ${ }^{1}$. 
Hasta años muy recientes el mundo del teatro ha vivido con una serie de dependencias propias de su misma organización empresarial, que determinaba en gran medida el desarrollo de la producción escénica. Y no sólo eso, sino la entidad de la obra dramática. El proceso de creación de ésta, y el de sus personajes, no sólo ha dependido de la imaginación del autor, sino de las circunstancias que rodeaban a la compañía para la que escribía. De ahí que el galán, la dama o el gracioso se sometieran al tipo de intérpretes que los representaban. No se puede hablar, pues, de arquetipos de la comedia española sin advertir los perfiles de los actores que los encarnaban en el escenario, de los rasgos físicos que los adornaban, de su posición social y económica en la compañía. De manera que lo normal es que dichos personajes tuvieran características similares en todos y cada uno de los actores que los hicieran. Por eso podemos hablar de la arrogancia de los galanes, de la belleza de las damas, de la gravedad de los barbas, de la comicidad de los graciosos, etc. Esto sucedía así no sólo por circunstancias artísticas, que las había, sino por circunstancias económicas, tan dignas o más que las anteriores. No hemos de olvidar que la actividad escénica se ha basado a lo largo de los siglos en un adecuado rendimiento mercantil que debía sostener todo el andamiaje organizativo del negocio teatral. Así, las compañías se constituían en torno a unos nombres (y unos rostros) que respondieran de manera más o menos adecuada a determinados personajes. Los intérpretes, que hacían generalmente papeles apropiados a sus perfiles (personajes «que les fueran»), se veían favorecidos si asumían cometidos con rasgos escénicos parecidos a los suyos, de forma que se produjera entre unos y otros corrientes de identificación más o menos creíbles. De todos es conocido que actores como Enric Borrás se crecían ante personajes de gran dignidad social, y que, cuando les tocaba hacer tipos de menor entidad, se sentían más incómodos. Y qué decir de la identificación entre Cosme Pérez y Juan Rana, para algunos considerada como seligrosa para la propia personalidad del conocido cómico del Siglo de Oro.

Aun con todas las prevenciones de rigor, no parece difícil establecer una serie de arquetipos que se repiten con harta frecuencia en las comedias españolas ${ }^{2}$. Y tampoco, hacer un recorrido por la historia para establecer relaciones entre los personajes de la commedia dell'arte (los más concretos y fijados de la historia del teatro occidental) con los de la comedia española. Esto, que en literatura es todo lo discutible que queramos, en los escenarios no lo parece tanto. Basta repasar las listas de compañías para advertir que un actor, o una actriz, solían (y suelen) hacer personajes (bastante) parecidos. Es como si dichos caracteres tuvieran siempre el mismo contorno, pero que hubiera que rellenarlo de carne y color, empresa reservada al genio del intérprete. 
En ese repaso de compañías del pasado se advierten nombres que se repiten con insistencia en torno a personajes como el gracioso, el galán y la dama, a los que no es complicado añadir el viejo o barba, criada, soldado y estudiante. Esta reiteración se acentúa de manera principal con el gracioso. Estamos ante un personaje cuyos perfiles externos encajarían con un actor de no de muy buen talle, voz poco entonada y brillante, y andares nada esbeltos y nobles. Más bien sería un tipo bajo, mejor rechoncho, con caminar divertido y presto a tropezar, ronca garganta que se extrema al intentar cantar o entonar una vieja balada. Esto no quiere decir que, ocasionalmente, algún primer actor haya probado el placer de representar un gran gracioso, que cree escrito para él; mas no es lo común. El gracioso suele ser un actor cómico, que algún día pudo ser primero, acomodado a una edad indefinida que no alcance la posibilidad del galán, aunque sí haga verosímil el escarceo amoroso con la criada principal. Recordemos que no hay personajes más jerarquizados social y moralmente que los de la comedia española. El gracioso podría tener su génesis teatral, no literaria, en una mezcla entre el esperpéntico Pulchinella napolitano y el ingenioso Arlequín bergamesco, aunque apenas tenga la entidad de ninguno de ellos. Por eso es el gran invento de la comedia española, y el personaje que la define; tanto, que apenas se puede hablar del género en su amplio sentido hasta que no hubiere un gracioso que acompaña al galán, lo aconseja, inventa las peripecias más significativas de la acción, y, de alguna manera, realiza una actividad determinante para la resolución de la comedia.

Observados desde la rotundidad con que Clarín acompaña a Rosaura, Tello a don Alonso, Catalinón a don Juan, Turín a Liseo, Morón a don Diego, Cosme a don Manuel, etc., etc., los criados de Rueda son una broma. Por muy simpáticos que sean Boreas (Ymenea) o Pajares y Guiomar (Los engañados), son una broma. La comedia es comedia cuando a la sombra de los protagonistas crece la figura del gracioso, que no sólo acompaña, sino aconseja, organiza, inventa, fantasea, traza, ingenia, urde, calcula, concibe, improvisa, etc. La comedia es comedia cuando Lope de Vega sitúa al gracioso dentro de la estructura misma de la acción. Antes, son meros antecedentes.

Por consiguiente, el gracioso es un personaje más de la comedia, pero también pieza imprescindible en su dramaturgia. Como expliqué en otro lugar ${ }^{3}$, suele desempeñar el papel de ayudante en el hipotético esquema actancial de cualquier conflicto de capa y espada. Siempre es el colaborador esencial del deseo del galán, que no es otro (en la mayoría de las ocasiones) que conquistar a la dama. Es personaje esencial en el tejido dramático de la comedia pero también, y de ahí su riqueza, en el sistema 
paródico introducido inteligentemente por los poetas. La comedia descansa la mayor parte de su comicidad en el juego escénico del gracioso, y, dentro de él, en la carga burlesca que conlleva, que no es la exclusiva, pero sí la principal. No hay más que advertir sus patronímicos para verificar el sentido del humor que encierran sus nombres: Calabaza o Calabazas, Cabellera, Catalinón, Caramanchel, Mendo, Mengo, Clarín, Clarindo, Chacón, Tello, Tristán, Turín, Capricho, Patín, Talón, Patacón, Mosquito, Candil, Espolín, Moscatel o Dinero, todos ellos fáciles de advertir reiterados en repartos de distintas comedias. A veces son auténticos apellidos, como Mendoza, Beltrán, Marín o Carrillo; o nombres propios, como Cosme, Pedro, Martín, Hernando, Roque, Roberto, Ginés, Esteban o Lorenzo. Sin duda alguna, entre las muchas señas de identidad que disponía la representación de la comedia, el nombre que designaba a los graciosos es una de las principales. Y, junto a él, su propia catadura física: estatura, voz, ademanes y andares. ¡Cuántos codazos de advertencia habrán visto los corrales de comedia cuando apareciera el gracioso en escena! «iAhí está!». Su presencia corrobora sin duda que el espectador se encontraba ante lo que quería: una comedia con sus lances, sus partes, sus amores, sus bailes..., y su gracioso.

\section{Algunos actores-graciosos célebres contemporáneos}

Decía antes que no había más que repasar los repartos de estrenos o reposiciones de comedias del Siglo de Oro para ratificar la imagen que este tipo de personaje tenía. Bien podemos comprobar que actores como José Franco, en una primera etapa, y Miguel Ángel, después, fueron graciosos del Teatro Español en el período que fue dirigido por Cayetano Luca de Tena. También Antonio Riquelme ${ }^{4}$ asumió algunos de tales papeles por esos años. Así mismo, encontramos después a Antonio Ferrandis como gracioso en algunos de los montajes de clásicos, pocos, que José Luis Alonso llevó a cabo en el Nacional María Guerrero. Hemos de recordar que mientras el Español sí mantuvo más exactamente una dedicación al teatro del Siglo de Oro, el María Guerrero, con Luis Escobar primero, y Alonso después, sólo acudía a ellos de manera ocasional ${ }^{5}$. De ahí que José Franco y Miguel Ángel ${ }^{6}$ fueran graciosos más constantes, como constante era un tipo de compañía que podría recordar en sus escalones artísticos y sociales a los antiguos elencos de antes de la guerra. Ferrandis, y José Morales, José Luis Heredia o Alfonso del Real, eran actores graciosos, pero no graciosos en el sentido de personajes que sólo esporádicamente (algunos, ni eso) interpretaron en su carrera. 


\section{Norma y ruptura en el personaje del Gracioso}

Un breve repaso a las condiciones físicas de todos estos intérpretes nos revela innatas cualidades humorísticas. José Franco era gordo y bajo, con aspecto melifluo y voz cantarina. Eficaz intérprete, asumió también algunas direcciones escénicas, recordándose como cómico nato. Su voz fue característica en los teatros radiofónicos de Radio Nacional de España. Miguel Ángel, bajo de estatura también, tenía un aspecto bastante distinto del anterior pues su primera impresión era equívoca y desagradable. No pocos papeles de «malo» asumió en producciones teatrales y cinematográficas de los años cincuenta. En cambio, manejaba a la perfección resortes de humor propios del gracioso. No fue difícil verlo en escenarios nacionales en papeles de ese sesgo, desde su llegada de los teatros universitarios, en los que probablemente también los representó. De Antonio Ferrandis poco hay que añadir a su conocido y famoso final de carrera profesional. Antes de llegar a la popularidad a través de series de televisión, fue miembro característico y fijo de la Compañía Nacional María Guerrero, uno de los preferidos de José Luis Alonso, aunque generalmente a la sombra de José Bódalo, el primer actor de aquel elenco. Sin ser exactamente un gracioso tópico, Ferrandis ${ }^{7}$ se distinguía por una buena vis cómica, que le hizo asumir con toda propiedad el papel de Cosme en La dama duende (1966), uno de los graciosos más protagonistas del Siglo de Oro español.

Hay que insistir en que el moderno sistema de producción teatral relativiza de manera extraordinaria el juego escénico de los actores. Después de la guerra civil es muy difícil hablar de graciosos en una compañía española; después de 1975, imposible. La razón no puede ser más sencilla e inmediata: no existe el concepto de repertorio ${ }^{8}$. Incluso en la Compañía Nacional de Teatro Clásico, creada en 1986, es curioso comprobar que no se han diseñado unos tipos especiales para unos actores especiales. No pocas veces se quejó Adolfo Marsillach, su primer director y quien más tiempo permaneció al frente de esa empresa, de la dificultad de mantener a los actores en la compañía debido a la imposible competencia con otros medios (cine y televisión principalmente). En esos primeros años el gracioso de la Compañía era Paco Portes, actor de extraordinario talante cómico, dúctil y eficaz, adecuado para estos papeles, que siempre quiso alternar con otros de más calado dramático. Portes fue el gracioso de El médico de su honra (1986) y Los locos de Valencia (1986), los dos primeros montajes de Marsillach en esta compañía, así como de Antes de todo es mi dama (1988). Después, la compañía experimentó continuos cambios, asumiendo ese papel actores tan distintos como Jesús Bonilla (El vergonzoso en palacio), Fernando Conde (La dama duende, El caballero de Olmedo, No hay burlas con el amor), Arturo Querejeta (La vida 


\section{César Oliva}

es sueño) y Héctor Colomé (La gran sultana, Don Gil de las calzas verdes y en la reposición de $E l$ médico de su honra). Como se puede comprobar, no todos dirigidos por el citado Marsillach. Bien podríamos decir que Portes y Colomé han sido los últimos graciosos de una compañía española dedicado a los clásicos ${ }^{9}$.

\section{Identidad y variedad en los graciosos áureos}

Por todo lo dicho hasta ahora es fácil suponer que los graciosos responden a un esquema bastante cerrado como personaje dramático. Lo cual es verdad sólo hasta cierto punto. Repasando todos y cada uno de los graciosos de la comedia española no tenemos por menos que aceptar que un actor podría aplicar recursos muy similares a la mayoría de los personajes que incluyera en su repertorio, pero eso no significa que fueran iguales. Si repasamos unos cuantos, incluidos dentro del canon de los más representados y vistos en los escenarios contemporáneos, sólo con algo más de una docena de ejemplos podemos advertir diferencias si no apreciables sí enriquecedoras. Es cierto que tan somero número bastaría para definir el gracioso clásico, es decir, el que acompaña al señor, lo ayuda, seduce a la criada siempre a la sombra de aquél, ingenia las peripecias más determinantes para el transcurso de la acción y, finalmente, ocupa un discreto pero notable segundo plano a la hora de las despedidas. Pero no es menos cierto que bastantes de esos graciosos adquieren tonalidades tan especiales que los hacen únicos dentro de su campo significante. Dentro del primer esquema tenemos, entre otros, a Tello ( $E l$ caballero de Olmedo), Pedro (La dama boba), Beltrán (El acero de Madrid), Carrillo (Los melindres de Belisa), Cabellera (Don Lucas del Cigarral), representantes de la mayoría de las parejas gracioso-criada que pueblan las comedias españolas. Pero merece la pena detenerse en esos otros que, bajo apariencia de modelo, esconden interesantes recovecos en su actividad escénica.

Empezando por Catalinón, en El Burlador de Sevilla, advertimos que éste no recoge ninguna de las migajas amorosas que desprende don Juan, lo que no deja de ser curioso. Siempre hay en los graciosos una cierta inclinación a la seducción, sea con criadas o esclavas. Pero Catalinón, como si quisiera predicar con el ejemplo una férrea oposición a la promiscuidad erótica de su amo, no tiene contacto con hembra ni, al parecer, quiere tenerlo. Todo lo contrario sucede con Moscatel, en No hay burlas con el amor, uno de los graciosos más interesantes del teatro áureo. En contra de la norma, que concede el poder seductor al galán y sólo su reflejo al 
criado, en esta comedia Calderón propone una curiosa inversión de valores, que deja bien clara en los primeros versos:

MOSCATEL. Que se ha trocado la suerte
al paso, pues siempre dio
el teatro, enamorado
al amo, y libre al criado.
No tengo la culpa yo
desta mudanza; y así
deja que hoy el mundo vea
esta novedad, y sea
yo el galán, tú el libre.

Este paródico arranque de comedia no impedirá que finalmente los galanes terminen sus vidas de solteros con las dos hermanas protagonistas, y el sufrido Moscatel con su pretendida Inés.

Otros graciosos equívocos son los que sirven a amos que resultan ser amas, es decir, damas disfrazadas de galán. Obsérvese que, en la comedia española, cuando una chica se disfraza de hombre lo hace con todas las consecuencias. Por eso tiene que adoptar a un criado-varón que demuestre con su presencia no sólo que la chica intenta hacerse pasar por chico, sino que el papel que representa en la comedia precisa estar acompañada por un gracioso. Sin él no sería galán, aun disfrazado. Dos son los grandes ejemplos del repertorio, Clarín (La vida es sueño) y Caramanchel (Don Gil de las calzas verdes), aunque hay bastantes más: de Tirso de Molina encontramos abundantes ejemplos, no obstante fue el poeta que más veces disfrazó a sus damas de galanes. Por ejemplo, en La huerta de Juan Fernández, en la que no sólo hace hombre a doña Petronila, la primera dama, sino a su criada, Tomasa, a la que convierte en criado; El amor médico, con doña Jerónima disfrazada de médico; La mujer por fuerza, en donde el criado Fabio sabe que su ama, Finea, es mujer pese a que aparezca como hombre; doña Ana y Boceguillas en Bellaco sois, Gó$m e z$, etc.; e incluso los acompañantes de Dinarda, Bernardo y Fabio, en El anzuelo de Fenisa, que permanecen ignorantes del sexo de su acompañante y que, en cierto modo, asumen un papel de graciosos todavía sin conformar. Más adelante volveremos sobre esta comedia que tiene aspectos bastante más curiosos de lo que parece. Clarín se distingue de Caramanchel en que sabe que sirve a una mujer (Rosaura) aunque aparezca vestida de hombre. La acompaña en la aventura de la joven despechada. En cambio el gracioso tirsiano tiene en su haber que ignora la condición de su dueña, que lo toma como sirviente a la entrada de Ma- 
drid («desde hoy te recibo/ en mi servicio», le dice doña Juana disfrazada de don Gil). La situación es de todo punto divertida puesto que Caramanchel no deja de sospechar del sexo de su ama. Es tan normal que advierta alguna anomalía (recordemos que doña Juana debe ser la primera actriz de la compañía y, por ende, la más atractiva), que al principio la llama, primero "paje», y después "capón», sobre todo desde que conoce el supuesto nombre de don Gil:

CARAMANCHEL. Capón sois hasta en el nombre, pues si en ello se repara, las barbas son en la cara lo mismo que el sobrenombre.

De ahí que no pueda en toda la comedia descubrir la condición de don Gil. Al final, incluso, cuando doña Juana se desvela como tal, continúan las dudas del criado:

CARAMANCHEL. ¿Y sois hombre, o sois mujer?

Otros graciosos, en cambio, son más difíciles de precisar por esos rasgos tópicos que no aparecen siempre de manera tan rotunda. En El anzuelo de Fenisa, el papel de gracioso parece reservado a Tristán, ayudante y consejero de Lucindo. Cuando llega a Sicilia acompañando a su amo, vemos cómo le previene del peligro de las mujeres que se le acercan tan sólo desembarcar. Tristán asume, ante todo, el papel de mentor, como cualquier gracioso al uso, aunque para ello le falten notas evidentes en su comportamiento y función como personaje. Por ejemplo, apenas tiene otro cometido que el de ser consejero, ignorando el de seductor de criadas, en este caso, Celia, con la que apenas mantiene otra relación que enterarse de la trastienda de Fenisa, sobre la que sospecha las peores intenciones para Lucindo. Por eso ni uno ni otro, amo y criado, están en la conclusión de esta extraña comedia. Salen hacia Valencia bastante antes de su fin, cuando recuperan parte de los bienes engañados por la meretriz a su cándido amo. No es casualidad que la obra la termine otro criado, Camilo, del galán Albano, en cuyo texto tampoco figuran rasgos de comicidad que lo delaten como gracioso. La parte cómica de la obra corresponde curiosamente a los dos acompañantes de Dinarda, Bernardo y Fabio, españoles que desembarcan en Palermo, junto a ella, y con idénticas ansias de aventuras. A ellos competen las escenas más cargadas de humor, como la del interrogatorio a que los somete Albano acerca de la condición de Dinardo (Dinarda). No sólo le responden en italiano, sino que Fabio se permite cantar una «canzone siciliana». Esa ambigüedad de caracteres, esa indefinición de los personajes cómicos, me hicieron pen- 


\section{Norma y ruptura en el personaje del Gracioso}

sar en que El anzuelo de Fenisa podría ser comedia anterior a la fijación expresa del papel de gracioso en el teatro de Lope de Vega ${ }^{10}$. Aunque se cite en la segunda impresión de $E l$ peregrino en su patria (1618), y su primera edición sea de 1617, nada hace afirmar que no proceda de antes, o que corresponda a una revisión de un primitivo original. Hay demasiadas indefiniciones en los papeles, incluidos los galanes, pero sobre todo en los criados.

Pero tampoco tiene tanta importancia esta imprecisión, si advertimos otras comedias con graciosos equívocos que llenan de conjeturas acerca de su redacción obras más tardías, como es el caso de El encanto sin encanto, de Calderón, sin duda posterior a La dama duende. Hay en ella un gracioso aparente, Franchipán, que, aunque así no sea designado por el autor (lo llama «criado»), no cabe duda de que se acerca bastante al modelo. También otro criado, Fabio, («viejo» en el texto) podría contener algunos elementos de gracioso, aunque bastantes menos que el anterior. Sirve éste a Serafina, la primera dama, mientras que aquél lo hace a Enrique, galán español que asume el rol de protagonista. Ni uno ni otro criado parecen ser, en el pleno sentido del término, graciosos, sobre todo si los comparamos con sus dos grandes precedentes en este tipo de comedias de magia y fantasía, como son Cosme y Candil, graciosos así mencionados de manera explícita en La dama duende y El galán fantasma. Con ciertas reservas que veremos a continuación, éstos cumplen de manera adecuada el papel de gracioso, pero ¿lo hacen Franchipán o Fabio, en comedia que se supone más retórica y reiterativa, a la vez que menos original, que las anteriores? Franchipán ayuda a su señor en todo, busca medios para salir de Marsella a Mesina (confiesa que ha sisado dinero a su amo, quehacer propio de gracioso, del que Cosme es maestro y directo precedente), tiene ganas de comer y beber hasta en las condiciones más difíciles, muestra todo el miedo del mundo cuando es encarcelado con Enrique en esa misteriosa torre de la que hará gala de atrevimiento Serafina y, además, se llama nada menos que Franchipán, nombre con carga grotesca evidente. Y por si faltara algo, requiebra a Libia al final de la comedia, que le contesta de manera ambigua aun reconociendo que lo acepta como pareja:

FRANCHIPÁN. Señora Libia, sepamos qué habemos de hacer del moro.

LIBIA. Trocarle por un cristiano.

Cristiano que es él, y moro un rebuscado motivo que inventó la criada para sacar con buen aire de un conflicto a su ama, Serafina. No cabe 


\section{César Oliva}

duda, pues, que hay graciosos como Franchipán que no aparecen como tales en el texto, pero cuya función corresponde con enorme exactitud al canon. Fabio podría ser otro gracioso si no se constituyera en un curioso servidor de todas las órdenes de su ama, Serafina, a la que obedece sin pestañear sea cual sea la insensatez del mandado. Fabio (calificado como «viejo» en el texto, como dijimos antes) es de ese tipo de personajes cuyo texto apenas deja entrever más carácter que el tópico de criado, pero cuya actividad escénica propicia una originalidad más allá de lo común. No es fácil advertir a un sirviente que cumpla más a rajatabla las indicaciones de su dueña o ama, a no ser que le tenga una admiración superior de lo normal. Al personaje se lo oye decir: «Dices bien», «Y así, iré a que luego al punto/ cuanto importe se prevenga», «Volando iré», «Yo lo intentaré señora», "Tú verás cómo te sirvo», además de prestarse de misteriosa voz en la no menos misteriosa torre a la que conduce a los españoles presos.

Decíamos antes que Cosme (La dama duende) podría ser uno de los máximos representantes del gracioso áureo. Y, en efecto, lo es. Llamado así por el autor, y expresado su comportamiento dentro de la norma del personaje (consejero, sisador, borrachín, simpático, olvidadizo, miedoso) la ausencia de uno de sus rasgos principales le da una especial personalidad: no mantiene relaciones eróticas con su homólogo femenino, la criada Isabel. Y eso que comparten un número de escenas superior a las que otras comedias dedican a los criados, y, además, de las más chispeantes del teatro clásico. Recordemos la escena central de la Jornada II, en la que Isabel vuelve a la habitación a poner la ropa blanca en un azafate, y se encuentra con el inesperado regreso de Cosme. No hay ninguna alusión erótica del gracioso hacia ella, de manera que tampoco sorprende que, al final, cuando la comedia termina con el compromiso matrimonial de don Manuel con doña Ángela, y de don Juan con Beatriz, diga el galán:

COSME.

DON MANUEL.

COSME.

DON MANUEL. COSME.
¡Gracias a Dios que ya el duende se declaró! Dime: ¿estaba borracho?

Si no lo estás, hoy con Isabel te casas. Para estarlo fuera eso, mas no puedo.

¿Por qué causa?

Por no malograr el tiempo que en estas cosas se gasta, pudiéndolo aprovechar 
en pedir de nuestras faltas

perdón, y humilde el autor

os le pide a vuestras plantas.

He aquí, pues, un gracioso que termina comedia, que tiene en ella un papel principalísimo (tanto o más texto que el galán), pero que no admite comprometerse con la criada. ¿Ingenio del poeta? ¿Imposición no menos genial del actor? Sea lo que fuere, en la historia de la literatura queda como modelo de irónico equívoco.

En El galán fantasma, Candil es criado de Astolfo, con cuyo nombre juega irónicamente el poeta:

CANDIL. Viendo que yo soy Candil, de mí alumbrarte has querido.

Y así oye cuanto pasa, si a callarlo te reduces; porque quiero hacer dos luces a la calle y a la casa.

Como su hermano mayor, Cosme, ayuda a su amo, colabora en la complicación de la trama, y aunque tiene sus escarceos con las criadas Lucrecia y Porcia (nombres burlones donde los haya) tampoco termina ligado a mujer alguna, y por parecidas razones a las anteriores. Después de asistir a los arreglos matrimoniales de rigor, el criado cierra la comedia:

CANDIL. Yo, que pasé tantos sustos, no quiero de nadie nada, sino de los mosqueteros el perdón de nuestras faltas, para que con esto fin demos al Galán Fantasma.

Que el gracioso se llame significativamente de una manera determinada añade rasgos de humor con su propio nombre, tal y como demuestra Candil, y tal y como demuestra Cabellera, en Entre bobos anda el juego, de Rojas Zorrilla.

DOÑA ISABEL. ¡Qué mal nombre!

CABELLERA.

Pues yo sé

que a todo calvo aficiona. 
Si el criado de don Lucas tiene abundante caballera es por contrastar no sólo con la ironía del propio poeta, Rojas Zorrilla, célebre por su calvicie, sino con la condición del amo, don Lucas, retratado por Cabellera como:

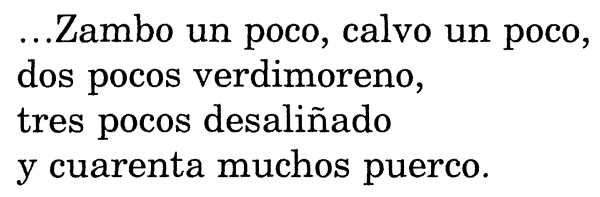

Comedia ésta en la que la comicidad del gracioso, evidente, no deja atrás ni mucho menos la del protagonista, don Lucas, que, a pesar de ser caballero, posee todas las cualidades grotescas posibles. Pocos galanes como éste, junto al don Diego moretiano, rivalizan en comicidad con los graciosos, estableciendo con ellos una curiosa y divertida disputa de roles. Don Lucas termina su comedia, y Mosquito, el criado de don Diego, la suya. No es extraño que el gracioso cierre las comedias. Con toda seguridad debía obedecer a la enorme simpatía que establecía esta figura del donaire con el público, que gustoso recibía las últimas palabras de la obra en su boca. Incluso en asuntos escabrosos, como en La Estrella de Sevilla, es quien remata la acción:

CLARINDO.

Y aquí

esta tragedia os consagra

Lope, dando así a La Estrella

de Sevilla eterna fama,

cuyo prodigioso caso

inmortales bronces guardan.

Otros conocidos graciosos que acaban la comedia, entre una larguísima lista, son el citado Mosquito (tanto el de El lindo don Diego, de Moreto, como el de El escondido y la tapada, de Calderón), Hernando (Los milagros del desprecio, de Lope), Batín (El castigo sin venganza, de Lope), Berrueco (Los novios de Hornachuelos, de Lope), Patín (Mujer, llora y vencerás, de Calderón, comedia en la que explícitamente se cita un segundo gracioso: Talón), Chacón (Dar tiempo al tiempo, de Calderón), y Patacón (Las manos blancas no ofenden, de Calderón). Entre ellos son curiosos los casos de Berrueco y Chacón. El primero, rizando el rizo del tópico final, prefiere descasarse (sensu recto) que casarse:

BERRUECO. Señor, pues hoy se descasan, yo te pido... 
Norma y ruptura en el personaje del Gracioso

\begin{tabular}{lc} 
MARINA. & \multicolumn{1}{c}{ Yo te ruego... } \\
$\begin{array}{l}\text { BERRUECO. } \\
\text { MARINA. }\end{array}$ & ... me descases. \\
BERRUECO. & \multicolumn{1}{c}{... me descases. } \\
MARINA. & $\begin{array}{l}\text { Que en ver a Marina tiemblo. } \\
\text { he de morir..., y a Berrueco } \\
\text { aborrezco. }\end{array}$ \\
REY. & \multicolumn{1}{c}{ Yo os aparto. } \\
MARINA. & Contenta estoy. \\
BERRUECO. & \multicolumn{1}{c}{ Yo contento. }
\end{tabular}
dia:

El segundo, Chacón, hace ironía semejante sobre el final de su come-

CHACÓN. Todos quedan bien; mas yo

quedo sin casar más bien;

y pues que «dar tiempo al tiempo»

trocó el pesar en placer,

los defectos perdonad

de quien yace a vuestros pies.

En otra comedia con tintes de tragedia, El Caballero de Olmedo, el poeta deja al Rey el honor de las últimas palabras (como en El villano en su rincón), pero no impide que Tello tenga la parte más importante de la conclusión, pues, además de contar con pelos y señales la traición y muerte de su amo don Alonso, sirve de acusador a los que incitaron el crimen.

TELLO. Presentes están, señor.

Don Rodrigo es el primero

y don Fernando el segundo.

La galería de graciosos no se enriquece del todo en los dramas populares de honor, como Peribáñez y Fuenteovejuna, de Lope, o El Alcalde de Zalamea, de Calderón. Dichos personajes desaparecen aquí metamorfoseándose en aldeanos (o «labradores») que asumen cometidos con contenido humorístico, pero sin alardear de otras características que distinguían a los graciosos. Incluso en el drama calderoniano, ese cometido cómico está presente, sobre todo, en la pareja don Mendo-Nuño, hidalgo venido a menos y su criado. En ellos, y en la pareja Chispa y Rebolledo, acompañante y tropa propiamente dicha. Quizás por eso no deje de ser 
lógico que estas obras las concluyen los galanes protagonistas (el propio Peribáñez y Frondoso) las dos primeras, y Pedro Crespo, el alcalde, la segunda. Sin embargo, y para demostrar que en terrenos como el que pisamos no hay reglas ni recetas, otro conocido drama de honor, de textura similar a los anteriores, La serrana de la Vera, de Vélez de Guevara, sí dispone de un aldeano, Mingo, cuyo personaje viene definido por el autor como «gracioso». No obstante, no asume en absoluto el modelo habitual, sino que, para ser distinto, incluso corteja a Gila, la protagonista, cosa que no lo salva de ser atado por ella a un roble, en la Tercera Jornada, hasta que elija la manera de morir a sus manos. Como obra de solución tremenda, el poeta deja al Maestre de Calatrava las últimas palabras del drama.

\section{A modo de conclusión de urgencia}

Después de mucho tiempo de cotejar graciosos en los escenarios que se ven unos en otros, parece oportuno afirmar que, sin dudar del carácter unívoco del personaje en sí, todos ellos disponen de rasgos suficientes para que sus intérpretes puedan encontrar elementos bastantes para su distinción y diferenciación. Y ello es así no sólo por la personalidad del actor sino porque en la misma entidad del personaje creado por el poeta no es difícil advertir atributos suficientes para pensar en que los graciosos pueden caracterizarse entre ellos, a pesar del canon que los sitúa en el paradigma de la comedia. Una cosa es el arquetipo y otra la exuberancia de su variedad, de lo que, estamos seguros, el teatro clásico español ofrece ejemplos de continuo. Por razones de espacio sólo hemos mencionado algunos, la mayoría de ellos de obras conocidas en los escenarios españoles de los últimos tiempos. Pero ahí queda la inmensa riqueza de la comedia áurea para poder precisar con mayor rigor que el modelo del gracioso es, por fortuna, continuamente violado y dignificado por los propios poetas.

\section{Notas}

${ }^{1}$ Compañía Corral de Almagro. Estrenada en Almagro y otras ciudades españolas, en 1977, con Manuel Gallardo (don Juan), Guillermo Marín (don Gonzalo de Ulloa), Jesús Aristu (Rey) y por supuesto José Luis Heredia (Catalinón).

${ }^{2}$ Ruiz Ramón, ya en la primera edición de su Historia del teatro español, 1 (Desde sus orígenes hasta 1900) (1967), hacía relación de los principales personajes de la comedia es- 


\section{Norma y ruptura en el personaje del Gracioso}

pañola, deṡde el rey hasta el villano, pasando por supuesto por el gracioso. Lo importante de su aportación es reconocer que aunque hay un "fuera" del personaje que lo acerca al arquetipo, también existe un "dentro" que lo define en profundidad. La dualidad "rostro" y "máscara" lo aclara de manera definitiva. A este respecto hay que ver también el estudio de Juana José de Prades, Teoría sobre los personajes de la comedia nueva, Madrid, 1963.

3 "El honor como oponente al juego teatral de galanes y damas», Gestos 3, University of California, Irvine, 1987, pp. 41-51.

4 Antonio Riquelme (1896-1968) fue un gran cómico de los años treinta y cuarenta de la escena española. Comenzó como meritorio en la compañía de María Guerrero, siendo titular del Teatro de la Comedia más de veinte años. Su extraordinaria delgadez le hacía especialmente simpático, sobre todo en papeles característicos de andaluz o madrileño castizo. Después de la guerra civil, fue ganado por el cine consagrándose como uno de los secundarios de lujo de Cifesa, y sólo esporádicamente regresaba al teatro. Intervino en más de doscientas películas. No es difícil encontrarlo en muchas de tonalidad folklórica, a veces haciendo pareja con Manolo Morán, otro de los grandes característicos de la época, que, en contra de Riquelme, se distinguía por su gordura.

5 De estas compañías y sus correspondientes repertorios tratamos en Los teatros público en España 1939-1978, de Bernal, F. y Oliva, C. La Avispa, Madrid, 1996.

${ }^{6}$ José Franco fue el Tello de Las bizarrías de Belisa (1941) y el Cosme de La dama duende (1942), mientras que Miguel Ángel, el Mosquito de El lindo don Diego (1963), todas ellas dirigidas por Cayetano Luca de Tena.

7 Antonio Ferrandis (1921-2000) fue muy popular en la televisión gracias al papel de Chanquete en la serie Verano azul. Incluso interpretó la primera película española galardonada con el Oscar a la mejor producción extranjera: Volver a empezar (1983), de José Luis Garci, y precisamente en un papel dramático. Pero sus inicios fueron en el teatro, y en papeles cómicos y dramáticos, como el Julepe de La rosa de papel, de Valle-Inclán, que protagonizó en el famoso montaje de José Luis Alonso de 1967. Con este mismo director hizo también el Tristán de El anzuelo de Fenisa (1965).

8 Debemos citar el esfuerzo de Antonio Guirau Sena por mantener una compañía de teatro clásico en esos años que cito. Con el Pequeño Teatro de Madrid, lo mismo actuaba en provincias que en el exterior (Estados Unidos, Alemania, Francia), aunque nunca tuviera el reconocimiento por parte de la crítica. En dicha compañía, el actor que más veces ha interpretado papeles de gracioso (Cosme, Caramanchel, Hernando) ha sido Francisco Racionero, con cuya presencia se rompe un tanto el esquema antes anunciado. Racionero es de estatura normal, buen físico, delgado, características que lo acercarían al galán, si no fuera por su innata y saludable tendencia a la gracia y a la broma que traslada sin rubor al escenario. Su eficacia como actor le ha llevado a protagonizar series de televisión, en las que hace gala de esas condiciones para la comicidad. Junto a Racionero, José Caride, Luis Perezagua y Miguel Caiceo también han sido eficaces graciosos en producciones de los años setenta y ochenta.

9 Otro director que ha intentado mantener una compañía dedicada al Siglo de Oro ha sido Manuel Canseco, al que podemos unir el más reciente esfuerzo de Pepe Maya y Amaya Curiases con Zampanó, y el Teatro Corsario, de Valladolid, dirigido por Fernando Urdiales.

10 Así lo dije en "El anzuelo de Fenisa: del texto al espectáculo", en El escritor y la escena III (Estudios en honor de Francisco Ruiz Ramón), AITENSO, Universidad Autónoma de Ciudad Juárez, 1995. 